\title{
An Agricultural Sensor Data Sparse Representation and Recovery Method Based on Redundant Dictionary
}

\author{
Feng Liu*, Zhong Yang \\ College of informatics, Huazhong Agricultural University, \\ Wuhan, 430070, China \\ ${ }^{*}$ Corresponding author
}

Keywords: Agricultural Sensor Data, Sparse Representation, Redundant Dictionary

\begin{abstract}
The applications based on Internet of Things are widely used. In these applications, how to efficiently compressed wireless sensor data and recover them accurately is an important problem. In order to solve this problem, we design an agricultural sensor data sparse representation and recovery method based on redundant dictionary. We test our method on real sensor data set and the results show that the sensor data can be compressed and recovered accurately.
\end{abstract}

\section{Introduction}

With the development of Internet of Things (IoT), a lot of applications based on IoT are designed and implemented. Wireless sensor networks (WSN) is an important part of an IoT system, and how to efficiently compressed these sensor data and accurately recover them is an essential problem for these applications. In order to solve this problem, in this paper, we will design an agricultural sensor data sparse representation and recovery method based on redundant dictionary.

Redundant dictionary or Overcomplete dictionary is an effective tool to do production and classification work and is widely used in signal and image processing domain. Meng et al.[1] use redundant dictionary for face recognition. In order to address the image denosing problem, Elad et al. [2] employ sparse and redundant representations over learned dictionaries. There are also a lot of algorithms to find the sparse representation, such as OMP[3] and ORMP[4]. In order to solve the dictionary construction problem, Aharon et al.[5] propose the K-SVD algorithm for sparse representation and prove that this algorithm is practical on natural images by several experiments. Mairal et al. [6] propose an online optimization algorithm based on stochastic approximations to construct dictionary and this algorithm can be used to process large training data sets.

Even though redundant dictionary are widely used in image processing domain, it is seldom found in sensor data processing area. In this paper, we use redundant dictionary method in the agricultural sensor data processing domain to achieve the sparse representation for agricultural sensor data, and find efficient sensor data compressing and recovery method.

\section{The Sparse Representation Method}

Let $D$ represent a redundant dictionary, $S$ represent the original agricultural sensor data and the coefficients of the dictionary's atoms are denoted by a vector $\alpha$. We want to find a specific $\alpha$ which satisfies (1) [7].

$$
\alpha_{\text {opt }}=\underset{\alpha}{\arg \min }\|\alpha\|_{0}+\gamma\|S-D \alpha\|_{2}^{2}
$$

Let $\|\alpha\|_{0}$ represent the 0 -norm of vector $\alpha$, which means the number of non-zero entries in $\alpha$. The main idea of dictionary based sparse representation is to use the linear combination of a small number of columns in dictionary $D$ to represent the original data $S$. In other words, If the value of $\|\alpha\|_{0}$ is small enough, the vector $\alpha$ can be regarded as a sparse representation of the original data $S$. 
It is very hard to find the optimum $\alpha$ in equation (1), because it is a NP-hard problem to find the minimum value of $\|\alpha\|_{0}$. So, a lot of researchers use equation (2) [7] instead.

$$
\alpha_{\text {opt }}=\underset{\alpha}{\arg \min }\|\alpha\|_{1}+\gamma\|S-D \alpha\|_{2}^{2}
$$

In agricultural wireless sensor networks, we need to measure different types of environmental data such as temperature, solar radiation, soil moisture et al. So, we want to use spare representation method to compress these sensor data to improve the efficiency of these systems. According to our research, we found that these environmental data are changing continuously and are connected. So, we try to find a sparse representation method for these sensor data.

\section{Experimental results}

We choose Discrete Cosine Transform (DCT) redundant dictionary and the real sensor data (http://lcav.epfl.ch/cms/lang/en/pid/86035) as our test data set. In this data set, we choose 100 ambient temperature sensor data from pdg2008-meteo- 1 and a 100*400 DCT dictionary to test our method. To solve this problem, we use ORMP[4] algorithm.

We recover these sensor data by equation (3), $S^{\prime}$ denotes the recovered data.

$$
S^{\prime}=D^{*} \alpha
$$

$S p$ is used to denote the number non-zero entries in $\alpha$. Figure 1 shows the results of agricultural sensor data sparse representation based on DCT dictionary when $S p$ is 1, 21, 41, 61, and 81 respectively. From figure 1, we can see that when $S p=1$, we cannot recover these sensor data only with one coefficient. When $S p=21$, the result is close to the original sensor data. When $S p=41$, the result is nearer to the original data than $S p=21$. When $S p=61$ and $S p=81$, we can find that the results can fit the original sensor data perfectly.

We use the error rate $(e)$ to measure data recovery accuracy and $e$ is calculated by Equation (4)

$$
e=\frac{\sqrt{\sum_{i=1}^{N}\left(s_{i}^{\prime}-s_{i}\right)^{2}}}{\sqrt{\sum_{i=1}^{N} s_{i}^{2}}}
$$

In (4), $N$ denotes the number of entries in the sensor data vector $S . s_{i}, s_{i}^{\prime}$ represent an entry in original sensor data vector and recovery sensor data vector respectively. Figure 2 shows that the value of $e$ is decreasing dramatically with an increasing $S p$ at beginning. But when $S p$ reaches around 60, the value of $e$ remains almost the same even if $S p$ increases. So, we can draw a conclusion that the sparse rate of these sensor data in the DCT redundant dictionary is around 0.6.

In IoT applications, we can use this method to compress the sensor data and choose different redundant dictionary for different type of environmental data. By this way, we can save data storage which is very import for portable devices.

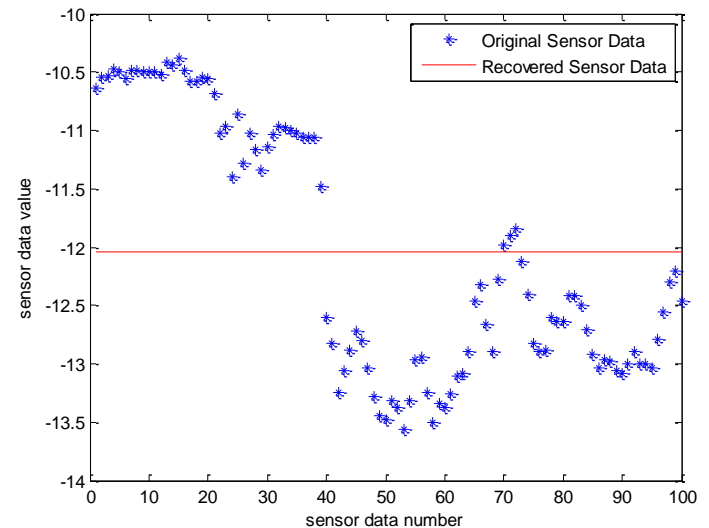

(a)

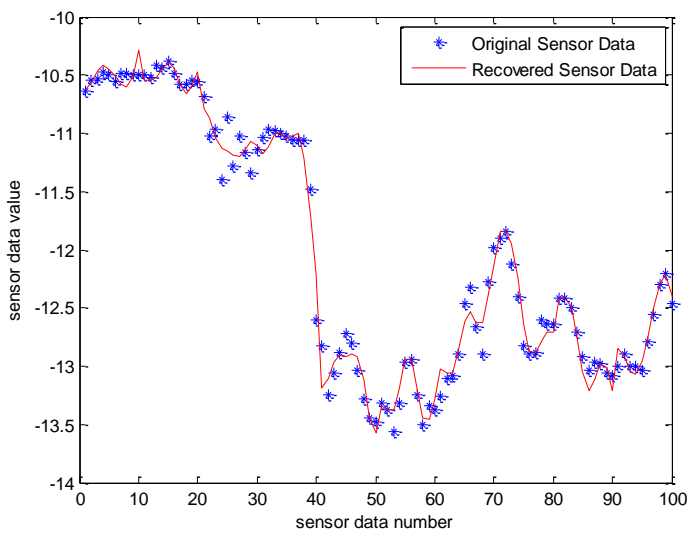

(b) 


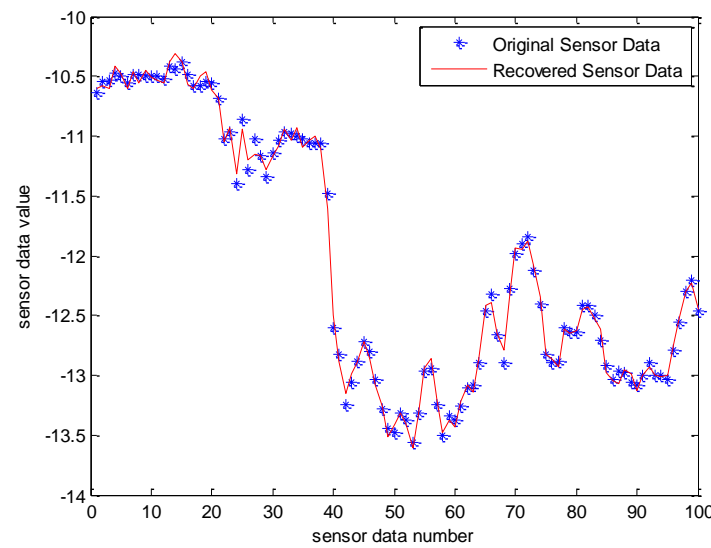

(c)

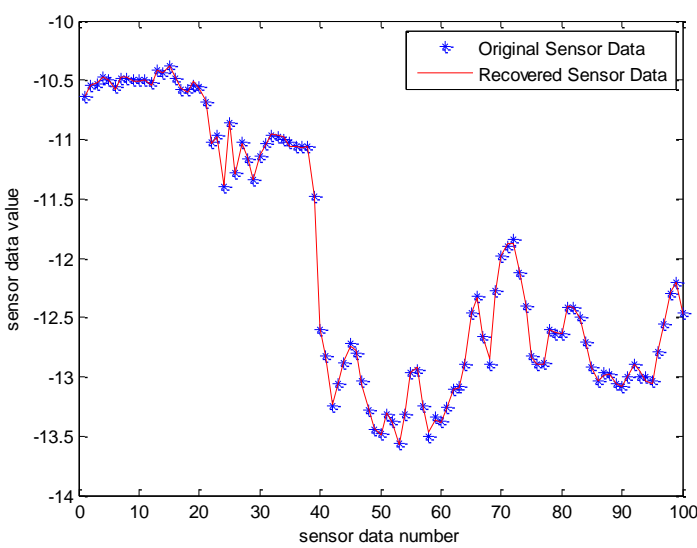

(d)

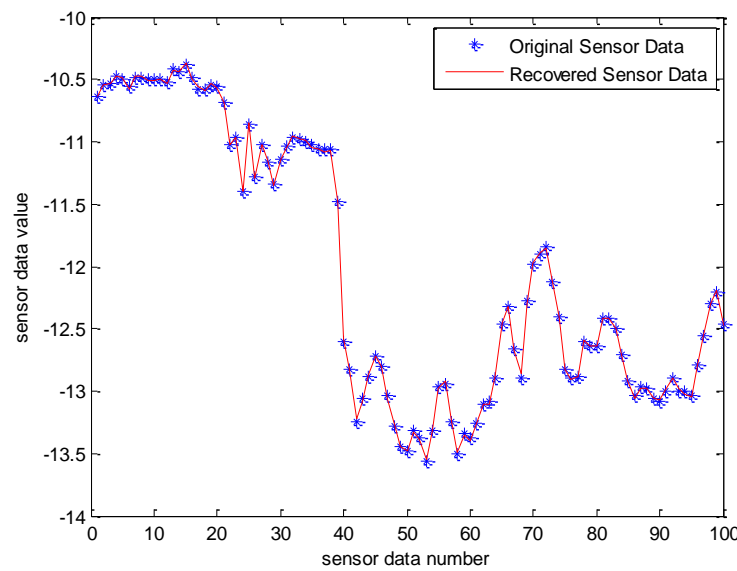

(e)

Figure 1 Agricultural Sensor Data (ambient temperature) Sparse Representation based on DCT

Dictionary (a) $S p=1$ （b) $S p=21$ (c) $S p=41$ （d） $S p=61$ (e ） $S p=81$

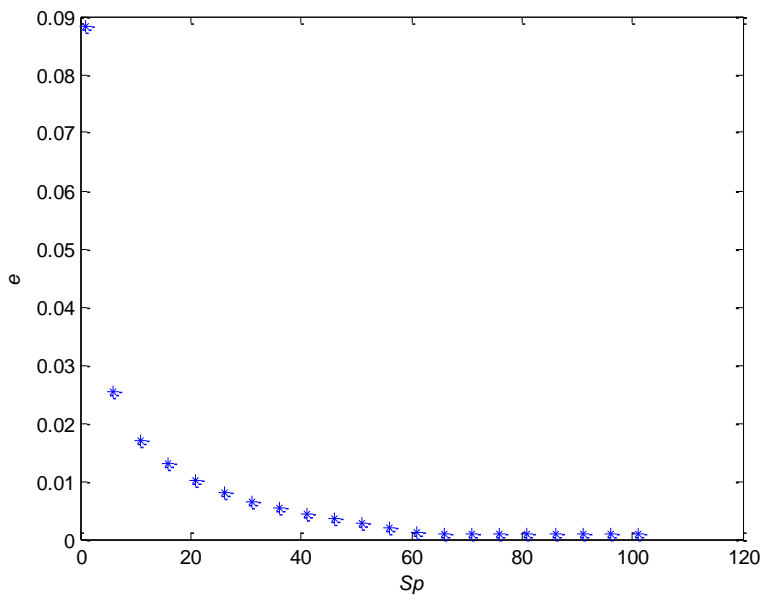

Figure 2 The error $(e)$ of recovered sensor data with different $S p$

\section{Summary}

In order to compress agricultural sensor data, in this paper we propose a sensor data sparse representation method based on DCT redundant dictionary. We test our method on the real sensor data set and find that these sensor data can be compressed and recovered accurately. Based on this study, in the future we can design efficient sensor data collection and storage method to save data storage space and furthermore, reduce energy consumption. 


\section{Acknowledgement}

This research was financially supported by Hubei Provincial Natural Science Foundation of China (Grant No. 2015CFB437), Specialized Research Fund for the Doctoral Program of Higher Education (Grant No. 20120146120002) and the Fundamental Research Funds for the Central Universities (Program No. 2013PY118).

\section{References}

[1] F. Meng, Z. Tang, Z. Wang, An improved redundant dictionary based on sparse representation for face recognition. Multimedia Tools and Applications, doi:10.1007/s11042-015-3083-6, 2015.

[2] M. Elad, M. Aharon, Image denoising via sparse and redundant representations over learned dictionaries, IEEE transactions on image processing, 15(12), 2006, pp.3736-3745.

[3] Y. Pati, R. Rezaiifar, P. Krislinaprasad. Orthogonal matching pursuit: Recursive function approximation with applications to wavelet decomposition. Proceedings of the 27th Annual Asilomar Conference on Signals, Sistems and Computers, 1993, pp.40-44.

[4] M. Gharavi-Alkhansari, T. S. Huang. A fast orthogonal matching pursuit algorithm. IEEE International Conference on Acoustics, Speech and Signal Processing, 1998,pp.1389-1392.

[5] M. Aharon, M. Elad, A. Brucksein, K-SVD: An Algorithm for Designing Overcomplete Dictionaries for Sparse Representation, IEEE Transactions on Signal Processing, 54(11), 2006, pp.4311-4322.

[6] J. Mairal, F. Bash, J. Ponce, G. Sapiro, Online Dictionary Learning for Space Coding, in proceedings of the $26^{\text {Th }}$ International conference on machine learning, 2009.

[7] Information on http://www.ux.uis.no/ karlsk/dle/ 